alterations in risk-assessment if molecular classification is added to conventional prognosticators.

Methodology Consecutive primary endometrial carcinoma patients diagnosed in 2016 were identified in participating centres. Original risk classification ('ORC16', ESGO/ESMO guidelines 2016) was compared to current molecular-based risk assessment ('MBR21', ESGO/ESMO guidelines 2021). Clinical and histopathological data were collected and tumor specimens were retrospectively submitted to PMS2, MSH6 and p53 immunohistochemistry and POLE mutation testing.

Result(s)* 226 patients were identified across five major European gynecologic oncology centres. Complete molecular and clinical data were available from 198 cases with a median follow-up time of 52.1 months. Median age was 64.9 years (30.9-90.9), 165 cases (83,3\%) were endometrioid histotype. Grade distribution included 85(42.9\%) G1, 63(31.8\%) G2, and 46(23.3\%) G3 tumors. 98(49.5\%) patients had stage IA disease, with the remaining stage IB $(n=51 ; 25.8 \%)$, stage II $(n=16 ; 8.0 \%)$, and stage III/IV $(n=33 ; 16.7 \%)$. Molecular classification yielded 43(21.7\%) MMR-D, 18(9.1\%) POLE, 43 (21.7\%) p53abn, and 94(47.5\%) p53wt tumors. If ORC16 was compared to MBR21, risk was found to be higher in 15 (7.6\%) cases, whereas $27(13.6 \%)$ cases were assigned to a lower risk group. No survival events were observed in the 9 patients where risk was changed from high-intermediate or high to low-risk.

Conclusion* We were able to demonstrate clinically relevant alterations of endometrial carcinoma risk assessment in a significant number of patients after adding TCGA-derived molecular data to conventional risk classification. Potential molecular-guided changes in patient management may help to avoid over- and undertreatment and will ultimately give rise to precision medicine strategies in endometrial carcinoma patient care.

\section{DEVELOPING AND VALIDATING ULTRASOUND-BASED RADIOMICS MODELS FOR PREDICTING HIGH-RISK ENDOMETRIAL CANCER}

${ }^{1} \mathrm{~F}$ Moro*, ${ }^{1} \mathrm{M}$ Albanese, ${ }^{2} \mathrm{~L}$ Boldrini, ${ }^{3} \mathrm{~V}$ Chiappa, ${ }^{2} \mathrm{~J}$ Lenkowicz, ${ }^{3} \mathrm{~F}$ Bertolina, ${ }^{1} \mathrm{~F}$ Mascilini, ${ }^{4} \mathrm{R}$ Moroni, ${ }^{2 ; 5} \mathrm{MA}$ Gambacorta, ${ }^{3} \mathrm{~F}$ Raspagliesi, ${ }^{1 ; 6} \mathrm{G}$ Scambia, ${ }^{1 ; 6} \mathrm{AC}$ Testa, ${ }^{1 ; 6} \mathrm{~F}$ Fanfani. ${ }^{1}$ Fondazione Policlinico Universitario Agostino Gemelli, IRCCS, Dipartimento Scienze della Salute della Donna, del Bambino e di Sanità Pubblica, Rome, Italy; ${ }^{2}$ Fondazione Policlinico Universitario Agostino Gemelli, IRCCS, Dipartimento di Diagnostica per Immagini, Radioterapia Oncologica ed Ematologia, UOC Radioterapia Oncologica, Rome, Italy; ${ }^{3}$ RCCS National Cancer Institute, Department of Gynecologic Oncology, Milan, Italy; ${ }^{4}$ Fondazione Policlinico Universitario Agostino Gemelli, IRCCS, Direzione Scientifica, Rome, Italy; ${ }^{5}$ Università Cattolica del Sacro Cuore, Istituto di Radiologia; ${ }^{6}$ Università Cattolica del Sacro Cuore, Dipartimento Scienze della Vita e Sanità pubblica, Rome, Italy

\subsection{6/ijgc-2021-ESGO.136}

Introduction/Background* Transvaginal ultrasound examination is the first imaging investigation for endometrial cancer. Ultrasound-based models for predicting high risk endometrial cancer have recently been published. However, none of these models includes radiomics features. Radiomics is an innovative high throughput technique extracting and translating high numbers of features from medical images into mineable data.

Aim of this study was to develop and validate ultrasoundbased radiomics models, aiming to differentiating high risk category, as defined by ESMO-ESGO-ESTRO in 2016, versus the remaining categories of risk.

Methodology This is a multicenter retrospective observational study. Patients with histologically confirmed diagnosis of endometrial cancer who had undergone preoperative ultrasound examination between 2016 and 2019 were identified from two centers. Patients recruited in Center 1 (Rome) were included as 'training set' $(n=396)$, while patients enrolled in Center 2 (Milan), as 'external validation set' $(n=102)$. Radiomics analysis was applied to the ultrasound images. Clinical

Abstract 387 Table 1 Logistic regression models for discriminating high risk group versus other three groups, and low risk group versus the other risk groups for validation set

\begin{tabular}{|c|c|c|c|c|c|c|c|c|c|c|c|c|c|c|}
\hline Predictive Models & $\begin{array}{l}\text { Optimal } \\
\text { cut-off }\end{array}$ & $\begin{array}{l}\text { AUC } \\
\text { estimate } \\
(95 \% \mathrm{Cl})\end{array}$ & $\begin{array}{l}\text { Sensitivity } \\
(\%) \\
(95 \% \mathrm{Cl})\end{array}$ & $\begin{array}{l}\text { Specificity } \\
(\%) \\
(95 \% \mathrm{Cl})\end{array}$ & $\begin{array}{l}\text { Accuracy } \\
(\%) \\
(95 \% \mathrm{Cl})\end{array}$ & $\begin{array}{l}\text { PPV } \\
(\%) \\
(95 \% \mathrm{Cl})\end{array}$ & $\begin{array}{l}\text { NPV } \\
(\%) \\
(95 \% \mathrm{Cl})\end{array}$ & $\begin{array}{l}\text { LR+ } \\
(95 \% \mathrm{Cl})\end{array}$ & $\begin{array}{l}\text { LR- } \\
(95 \% \mathrm{Cl})\end{array}$ & $\begin{array}{l}\mathrm{TP} \\
\text { (n) }\end{array}$ & $\begin{array}{l}\text { TN } \\
\text { (n) }\end{array}$ & $\begin{array}{l}\text { FN } \\
\text { (n) }\end{array}$ & $\begin{array}{l}\text { FP } \\
\text { (n) }\end{array}$ & $\begin{array}{l}\text { Total } \\
\text { (n) }\end{array}$ \\
\hline $\begin{array}{l}\text { Radiomics model to predict } \\
\text { low risk }\end{array}$ & 0.38 & $\begin{array}{l}0.71(0.61 \\
-0.81)\end{array}$ & $\begin{array}{l}65.0(49.6 \\
-78.3)\end{array}$ & $\begin{array}{l}64.5(52.2 \\
-75.5)\end{array}$ & $\begin{array}{l}64.7(55.1 \\
-73.5)\end{array}$ & $\begin{array}{l}54.2(40.2- \\
67.7)\end{array}$ & $\begin{array}{l}74.1(61.3 \\
-84.3)\end{array}$ & $\begin{array}{l}1.83(1.22 \\
-2.75)\end{array}$ & $\begin{array}{l}0.54(0.34 \\
-0.86)\end{array}$ & 26 & 40 & 14 & 22 & 102 \\
\hline $\begin{array}{l}\text { Clinical-ultrasound model to } \\
\text { predict low risk }\end{array}$ & 0.46 & $\begin{array}{l}0.85(0.78 \\
-0.92)\end{array}$ & $\begin{array}{l}70.0(54.7 \\
-82.4)\end{array}$ & $\begin{array}{l}80.6(69.5 \\
-88.9)\end{array}$ & $\begin{array}{l}76.4(67.0 \\
-84.3)\end{array}$ & $\begin{array}{l}70.0(54.7- \\
82.4)\end{array}$ & $\begin{array}{l}80.6(69.5 \\
-88.9)\end{array}$ & $\begin{array}{l}3.62(2.09 \\
-6.25)\end{array}$ & $\begin{array}{l}0.37(0.23 \\
-0.61)\end{array}$ & 28 & 50 & 12 & 12 & 102 \\
\hline $\begin{array}{l}\text { Mixed model to predict low } \\
\text { risk }\end{array}$ & 0.36 & $\begin{array}{l}0.85(0.80 \\
-0.93)\end{array}$ & $\begin{array}{l}87.5(74.7 \\
-95.0)\end{array}$ & $\begin{array}{l}72.5(60.6 \\
-82.4)\end{array}$ & $\begin{array}{l}78.4(69.1 \\
-85.9)\end{array}$ & $\begin{array}{l}67.3(53.8 \\
-78.8)\end{array}$ & $\begin{array}{l}90.0(79.4 \\
-96.0)\end{array}$ & $\begin{array}{l}3.19(2.09 \\
-4.86)\end{array}$ & $\begin{array}{l}0.17(0.0- \\
0.40)\end{array}$ & 35 & 45 & 5 & 17 & 102 \\
\hline $\begin{array}{l}\text { Radiomics model to predict } \\
\text { high risk }\end{array}$ & 0.41 & $\begin{array}{l}0.80(0.70 \\
-0.88)\end{array}$ & $\begin{array}{l}58.7(44.3 \\
-72.0)\end{array}$ & $\begin{array}{l}85.7(74.8 \\
-93.0)\end{array}$ & $\begin{array}{l}73.5(64.4 \\
-81.3)\end{array}$ & $\begin{array}{l}77.1(61.5 \\
-88.6)\end{array}$ & $\begin{array}{l}71.6(60.1- \\
81.4)\end{array}$ & $\begin{array}{l}4.10(2.12 \\
-7.96)\end{array}$ & $\begin{array}{l}0.48(0.33 \\
-0.71)\end{array}$ & 27 & 48 & 19 & 8 & 102 \\
\hline $\begin{array}{l}\text { Clinical-ultrasound model to } \\
\text { predict high risk }\end{array}$ & 0.40 & $\begin{array}{l}0.87(0.81 \\
-0.94)\end{array}$ & $\begin{array}{l}67.3(53.1 \\
-79.5)\end{array}$ & $\begin{array}{l}89.2(78.5 \\
-95.0)\end{array}$ & $\begin{array}{l}79.4(70.2 \\
-86.7)\end{array}$ & $\begin{array}{l}83.7(68.8 \\
-92.3)\end{array}$ & $\begin{array}{l}76.9(65.3- \\
85.4)\end{array}$ & $\begin{array}{l}6.29(2.88 \\
-14.0)\end{array}$ & $\begin{array}{l}0.37(0.24 \\
-0.56)\end{array}$ & 31 & 50 & 15 & 6 & 102 \\
\hline $\begin{array}{l}\text { Mixed model to predict high } \\
\text { risk }\end{array}$ & 0.42 & $\begin{array}{l}0.88(0.81 \\
-0.93)\end{array}$ & $\begin{array}{l}67.3(52.9 \\
-79.1)\end{array}$ & $\begin{array}{l}91.0(80.7 \\
-96.1)\end{array}$ & $\begin{array}{l}80.3(71.3 \\
-87.5)\end{array}$ & $\begin{array}{l}86.1(71.3 \\
-93.9)\end{array}$ & $\begin{array}{l}77.2(65.8 \\
-85.7)\end{array}$ & $\begin{array}{l}7.55(3.19 \\
-18.0)\end{array}$ & $\begin{array}{l}0.36(0.23 \\
-0.55)\end{array}$ & 31 & 51 & 15 & 5 & 102 \\
\hline
\end{tabular}

AUC, area under the receiver-operating characteristics curve; LR+, positive likelihood ratio; LR-, negative likelihood ratio; NPV, negative predictive value; PPV, positive predictive value; TN, true negatives; TP, true positives, FN, false negatives; FP, false positives. Radiomics model is a model generated from radiomics features. Clinical-ultrasound model is a model generated from clinical and ultrasound features. Mixed model is a model generated from clinical, ultrasound and radiomics features. 
(including preoperative biopsy), ultrasound and radiomics features that proved to be different at the univariate analysis on the training set were considered for multivariate analysis and for developing ultrasound-based machine learning assessment models.

Result(s)* For discriminating high risk category versus the other categories one random forest model from the radiomics features (radiomics model), one binary logistic regression model from clinical and ultrasound features (clinical-ultrasound model), and another binary logistic regression model from clinical, ultrasound and previously selected radiomics features (mixed model) were created.

In the validation set, the radiomics model for predicting high risk showed AUC 0.80, sensitivity 58.7\%, specificity $85.7 \%$, positive likelihood ratio $(\mathrm{LR}+) 4.10$ and negative likelihood ratio (LR-) 0.48 ; the clinical-ultrasound model showed AUC 0.87, sensitivity 67.3\%, specificity 89.2\%, LR+ 6.29 and LR- 0.37; and the mixed model showed AUC 0.88, sensitivity $67.3 \%$, specificity $91.0 \%, \mathrm{LR}+7.55$ and LR- 0.36 (table 1).

Conclusion* The mixed model including radiomics, clinical (including preoperative biopsy) and ultrasound features provided the best performance, even if the accuracy was slightly higher in comparison with the model based only on clinical and ultrasound variables. Interestingly, the model based only on radiomics features was able to provide good accuracy to discriminate high risk group versus the others.

\section{MOLECULAR PROFILING OF NSMP HIGH-RISK ENDOMETRIAL CANCERS OF THE PORTEC-3 TRIAL - PROGNOSTIC REFINEMENT AND DRUGGABLE TARGETS}

${ }^{1} \mathrm{~L}$ Vermij ${ }^{*},{ }^{2} \mathrm{M}$ Powell, ${ }^{1} \mathrm{~A}$ Leon-Castillo, ${ }^{3} \mathrm{~S}$ De Boer, ${ }^{4} \mathrm{~L}$ Mileshkin, ${ }^{5} \mathrm{H}$ Mackay, ${ }^{6} \mathrm{~A}$ Leary, ${ }^{7} \mathrm{HW}$ Nijman, ${ }^{8} \mathrm{~N}$ Singh, ${ }^{9} \mathrm{P}$ Pollock, ${ }^{10} \mathrm{P}$ Bessette, ${ }^{11} \mathrm{C}$ Haie-Meder, ${ }^{1} \mathrm{~V}$ Smit, ${ }^{12} \mathrm{R}$ Edmondson,

${ }^{12} \mathrm{E}$ Crosbie, ${ }^{3} \mathrm{R}$ Nout, ${ }^{3} \mathrm{~N}$ Horeweg, ${ }^{3} \mathrm{CL}$ Creutzberg, ${ }^{1 ; 13} \mathrm{~T}$ Bosse. ${ }^{1}$ Leiden University Medical Center (LUMC), Pathology, Leiden, Netherlands; ${ }^{2}$ Barts Health NHS Trust, Clinical Oncology, UK; ${ }^{3}$ Leiden University Medical Center (LUMC), Radiation Oncology, Leiden, Netherlands; ${ }^{4}$ Peter MacCallum Cancer Centre, Medical Oncology, Melbourne, Australia; ${ }^{5}$ Sunnybrook Health Sciences Centre -Odette Cancer Centre, Medical Oncology and Hematology, Toronto, Canada; ${ }^{6}$ Gustave Roussy, Medical Oncology, Villejuif, France; ${ }^{7}$ University Medical Center Groningen, Gynaecology, Groningen, Netherlands; ${ }^{8}$ Barts Health NHS Trust, Pathology, London, UK; ${ }^{9}$ QUT Gardens Point Campus, Institute of Health and Biomedical Innovation, Brisbane City, Australia; ${ }^{10}$ Université de Sherbrooke, Obstetrics and Gynaecology, Sherbrooke, Canada; ${ }^{11}$ Gustave Roussy, Radiation Oncology, Villejuif, France; ${ }^{12}$ St Mary's Hospital, Institute of Cancer Sciences, Manchester, UK; ${ }^{13}$ Leiden University Medical Center, Department of Pathology, Leiden, Netherlands

\subsection{6/ijgc-2021-ESGO.137}

Introduction/Background* The molecular endometrial cancer (EC) classification has proven prognostic value and can direct adjuvant treatment decisions. Despite this, a relatively large group of EC is still molecularly unclassified (NSMP-EC). In this study we aimed to identify biomarkers among high-risk NSMP-EC patients with prognostic and/or predictive relevance. Methodology Paraffin-embedded tumour material $(n=423)$ from the PORTEC-3 HREC trial were available for analysis. All patients with NSMP-EC were selected, hence those without pathogenic POLE mutations, mismatch repair deficiency
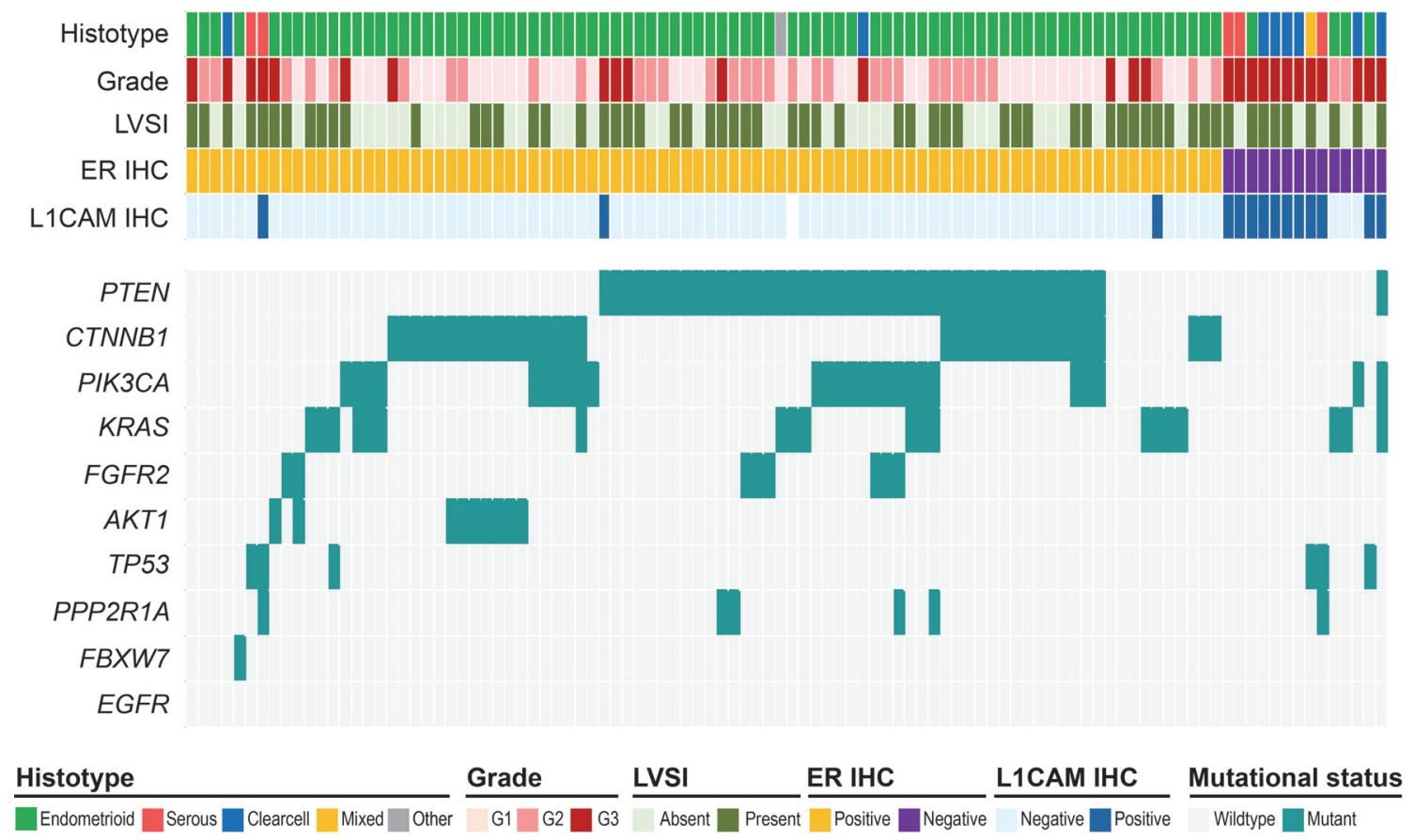

Abstract 397 Figure 1 Overview of clinicopathological and molecular characteristics of NSMP-EC from PORTEC-3 